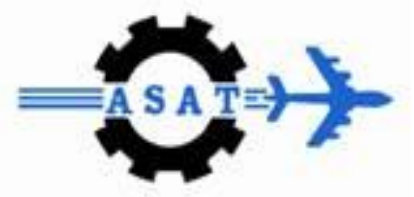

\title{
Peak to Average Power Ratio Reduction in Single Carrier OFDMA Systems
}

\author{
Mohamed Salah $^{*}$, Gamal Abdel-Fadeel ${ }^{* *}$, and Zaki B. Nossair ${ }^{* * *}$
}

\begin{abstract}
Single carrier frequency division multiple access (SC-FDMA) which utilizes single carrier modulation at the transmitter and frequency domain equalization at the receiver is a technique that has similar performance and essentially the same overall structure as those of an OFDMA system. One prominent advantage over OFDMA is that the SC-FDMA signal has lower peak-to-average power ratio (PAPR). SC-FDMA has drawn great attention as an attractive alternative to OFDMA, especially in the uplink communications where lower PAPR greatly benefits the mobile terminal in terms of the transmit power efficiency. SC-FDMA is currently a working assumption for the uplink multiple access scheme in 3GPP Long Term Evolution (LTE). To achieve low PAPR, a novel scheme, SC-OFDMA with super frame using intermediate mapping (SF-IFDMA), is proposed in this paper. In our proposed scheme, $\mathrm{B}$ frames with $\mathrm{N}$ subcarriers will be combined into one super frame, then SC-OFDM technique is applied to reduce the PAPR of the super frame, the super frame with lowered PAPR will finally be divided into B OFDM frames for the transmission. The combining and dividing process is equivalent to the concatenation process. The proposed scheme requires no side information and can significantly reduce PAPR while maintaining small number of subcarriers.
\end{abstract}

Keywords: Single carrier OFDMA with intermediate mapping (IFDMA), IFDMA with super frame (SF-IFDMA), peak to average power ratio (PAPR).

\section{Introduction}

As wireless multimedia applications become more widespread, demand for higher data rate is leading to utilization of a wider transmission bandwidth. With a wider transmission bandwidth, frequency selectivity of the channel becomes more severe and thus the problem of inter-symbol interference (ISI) becomes more serious. In a conventional single carrier communication system, time domain equalization in the form of tap delay line filtering is performed to eliminate ISI. However, in case of a wide band channel, the length of the time domain filter to perform equalization becomes prohibitively large since it linearly increases with the channel response length.

One way to mitigate the frequency-selective fading seen in a wide band channel is to use a multicarrier technique which subdivides the entire channel into smaller sub-bands, or subcarriers. Orthogonal frequency division multiplexing (OFDM) is a multicarrier modulation technique which uses orthogonal subcarriers to convey information. In the frequency domain,

\footnotetext{
* Teach. Assist., MTI University, Mohmmd_salah@yahoo.com

** Prof. Dr., Helwan University, Gam_hel@yahoo.com.

**** Associate Prof. Dr., Helwan University, Znossair@yahoo.com.
} 
since the bandwidth of a subcarrier is designed to be smaller than the coherence bandwidth, each sub-channel is seen as a flat fading channel which simplifies the channel equalization process. In the time domain, by splitting a high-rate data stream into a number of lower-rate data stream that are transmitted in parallel, OFDM resolves the problem of ISI in wide band communications [1]. But OFDM has its disadvantages: High peak-to-average power ratio (PAPR), high sensitivity to frequency offset, and a need for an adaptive or coded scheme to overcome spectral nulls in the channel [2], [3]. In this correspondence, we give an overview of a single carrier FDMA (SC-FDMA) system, which is a newly developed multiple access scheme adopted in the uplink of 3GPP Long Term Evolution (LTE), and show some research results on its PAPR characteristics.

The remainder of this paper is organized as follows: Section 2 gives an overview of SCFDMA. Section 3 presents our proposed scheme of SC-OFDMA with super frame. Simulation results will be provided in Section 4. We conclude our work at the end.

\section{Overview of SC-FDMA}

Figure 1 shows a block diagram of an SC-FDMA system. SC-FDMA can be regarded as DFT-spread orthogonal frequency division multiple access (OFDMA), where time domain data symbols are transformed to frequency domain by DFT before going through OFDMA modulation [4]. The orthogonality of the users stems from the fact that each user occupies different subcarriers in the frequency domain, similar to the case of OFDMA. Because the overall transmit signal is a single carrier signal, PAPR is inherently low compared to the case of OFDMA which produces a multicarrier signal [5].

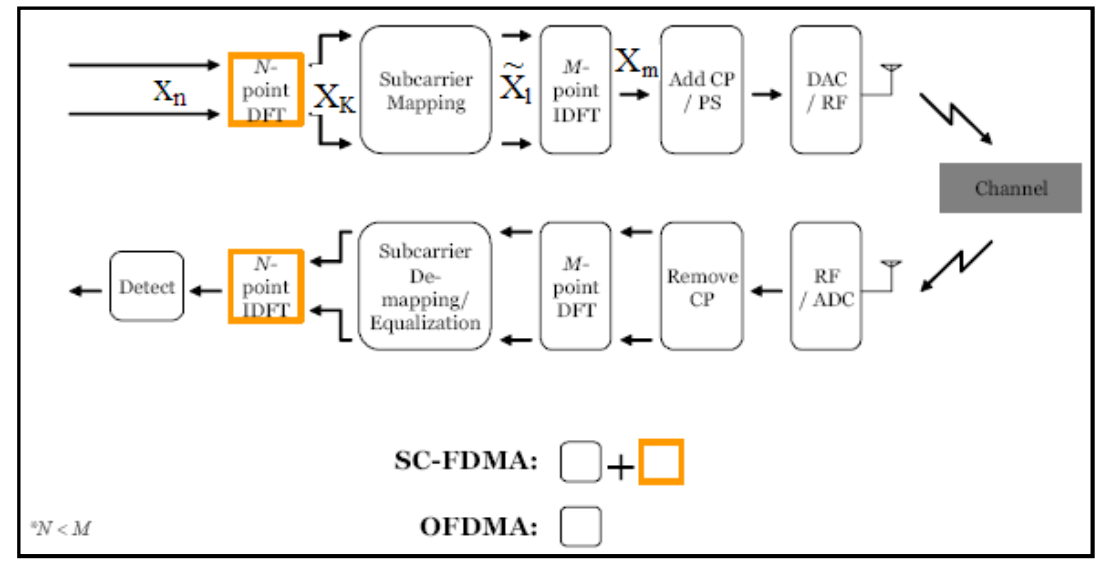

Fig. 1 A block diagram of an SC-FDMA system

The transmitter of an SC-FDMA system first groups the modulation symbols into blocks each containing $\mathrm{N}$ symbols. Next it performs an N-point DFT to produce a frequency domain representation of the input symbols. It then maps each of the N-DFT outputs to one of the $\mathrm{M}$ $(>\mathrm{N})$ orthogonal subcarriers that can be transmitted. If $\mathrm{N}=\mathrm{M} / \mathrm{Q}$ and all terminals transmit $\mathrm{N}$ symbols per block, the system can handle Q simultaneous transmissions without co-channel interference. $\mathrm{Q}$ is the bandwidth expansion factor of the symbol sequence. As in OFDMA, an M-point IDFT transforms the subcarrier amplitudes to a complex time domain signal.

The transmitter performs two other signal processing operations prior to transmission. It inserts a set of symbols referred to as a cyclic prefix $(\mathrm{CP})$ in order to provide a guard time to 
prevent inter-block interference (IBI) due to multipath propagation. The transmitter also performs a linear filtering operation referred to as pulse shaping in order to reduce out-ofband signal energy. In general, CP is a copy of the last part of the block, which is added at the start of each block for a couple of reasons. First, CP acts as a guard time between successive blocks. If the length of the $\mathrm{CP}$ is longer than the maximum delay spread of the channel, or roughly, the length of the channel impulse response, then, there is no IBI. Second, since CP is a copy of the last part of the block, it converts a discrete time linear convolution into a discrete time circular convolution. Thus transmitted data propagating through the channel can be modeled as a circular convolution between the channel impulse response and the transmitted data block, which in the frequency domain is a point-wise multiplication of the DFT frequency samples. Then, to remove the channel distortion, the DFT of the received signal can simply be divided by the DFT of the channel impulse response point-wise or a more sophisticated frequency domain equalization technique can be implemented.

The receiver transforms the received signal into the frequency domain via DFT, de-maps the subcarriers, and then performs frequency domain equalization. Most of the well known time domain equalization techniques, such as minimum mean square error (MMSE) equalization, decision feedback equalization (DFE), and turbo equalization, can be applied to the frequency domain equalization. The equalized symbols are transformed back to the time domain via IDFT, and detection and decoding take place in the time domain.

\subsection{Subcarrier Mapping}

There are two methods to choose the subcarriers for transmission; distributed subcarrier mapping and localized subcarrier mapping.

In the distributed subcarrier mapping mode, DFT outputs of the input data are allocated over the entire bandwidth with zeros occupying the unused subcarriers, whereas consecutive subcarriers are occupied by the DFT outputs of the input data in the localized subcarrier mapping mode. We will refer to the localized subcarrier mapping mode of SC-FDMA as localized FDMA (LFDMA) and distributed subcarrier mapping mode of SC-FDMA as distributed FDMA (DFDMA). The case of $\mathrm{M}=\mathrm{Q} \times \mathrm{N}$ for the distributed mode with equidistance between occupied subcarriers is called Interleaved FDMA (IFDMA) [6], [7]. An example of SC-FDMA subcarrier mappings in the frequency domain for $\mathrm{N}=4, \mathrm{Q}=3$ and $\mathrm{M}$ $=12$ is illustrated in Fig. 2 .

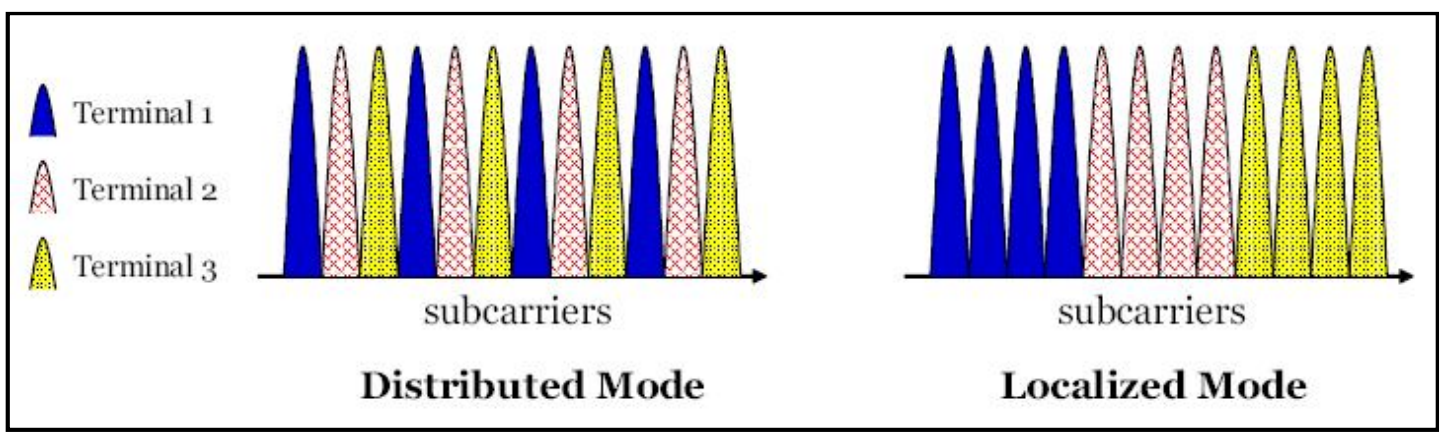

Fig. 2 Subcarrier allocation methods for multiple users $(Q=3$ users, $\mathrm{M}=12$ subcarriers, and $\mathrm{N}=4$ subcarriers allocated per user) 
Depending on the subcarrier mapping method, the SC-FDMA modulated symbols in the time domain differ [8]. For IFDMA, referring to Fig. 1 the frequency samples after subcarrier mapping $\widetilde{X}_{I}$ can be described as follows.

$\widetilde{X}_{\mathrm{l}}=\left\{\begin{array}{cc}\mathrm{X}_{\mathrm{l}} / \mathrm{Q} & , \mathrm{l}=\mathrm{Q} \cdot \mathrm{k}(0 \leq \mathrm{k} \leq \mathrm{N}-1) \\ 0 & , \text { otherwise }\end{array}\right.$

The time symbols $x_{m}$ which are obtained by taking inverse DFT of $\widetilde{X}_{1}$ will be:

Let $\mathrm{m}=\mathrm{N} . \mathrm{q}+\mathrm{n}$, where $0 \leq \mathrm{q} \leq \mathrm{Q}-1$ and $0 \leq \mathrm{n} \leq \mathrm{N}-1$ then,

$$
\begin{aligned}
\mathrm{x}_{\mathrm{m}}\left(=\mathrm{x}_{\mathrm{N} . \mathrm{q}+\mathrm{n}}\right) & =\frac{1}{\mathrm{M}} \sum_{\mathrm{l}=0}^{\mathrm{M}-1} \widetilde{\mathrm{X}}_{\mathrm{l}} \mathrm{e}^{\mathrm{j} 2 \pi \frac{\mathrm{m}}{\mathrm{M}} \mathrm{l}} \\
& =\frac{1}{\mathrm{Q}} \operatorname{IDFT}\left\{\mathrm{X}_{\mathrm{k}}\right\} \\
& =\frac{1}{\mathrm{Q}} \mathrm{x}_{\mathrm{n}}
\end{aligned}
$$

The resulting time symbols $\mathrm{x}_{\mathrm{m}}$ are simply a repetition of the original input symbols $\mathrm{x}_{\mathrm{n}}$ with a scaling factor of 1/Q and some phase rotation in the time domain [9]. Therefore, the PAPR of IFDMA signal is the same as in the case of conventional single carrier signal. DFDMA and LFDMA have the same time symbol structure; they have exact copies of input time symbols with a scaling factor of $1 / \mathrm{Q}$ in the N-multiple sample positions and in between values are sum of all the time input symbols in the input block with different complex weighting. Because of this, we can expect to see more fluctuation and higher peak in amplitude for DFDMA and LFDMA. Figure 3 shows the time symbols for different subcarrier mapping schemes. Figure 4 shows the amplitude of the signal for each subcarrier mapping for $M=64, N=16$, $\mathrm{Q}_{\text {IFDMA }}=4$, and $\mathrm{Q}_{\text {LFDMA, DFDMA }}=3$ without pulse shaping and we can see more fluctuation and higher peak for LFDMA and DFDMA.

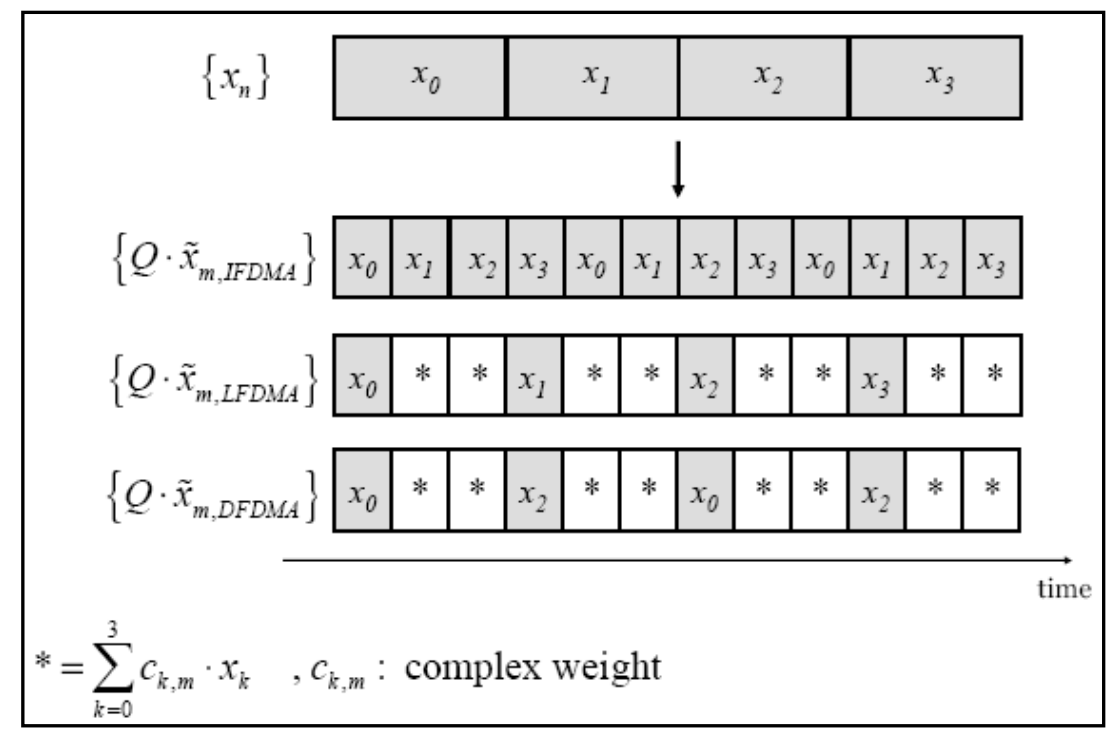

Fig. 3 Time symbols of different subcarrier mapping 


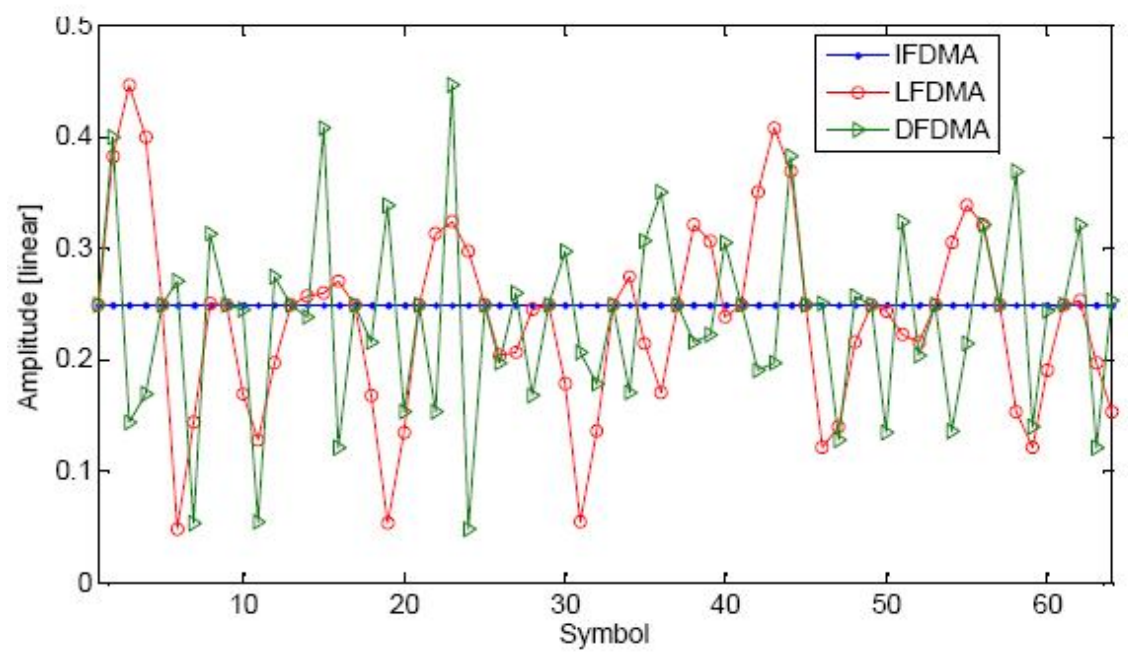

Fig. 4 Amplitude of SC-FDMA signals (no pulse shaping)

\subsection{SC-FDMA and OFDMA}

Figure 1 also shows a block diagram of an OFDMA transmitter. It has much in common with SC-FDMA. The only difference is the presence of the DFT in SC-FDMA. For this reason SCFDMA is sometimes referred to as DFT-spread or DFT- precoded OFDMA. Other similarities between the two include: Block-based data modulation and processing, division of the transmission bandwidth into narrower sub-bands, frequency domain channel equalization process, and the use of $\mathrm{CP}$.

However, there are distinct differences that make the two systems perform differently. In terms of data detection at the receiver, OFDMA performs it on a per subcarrier basis whereas SC-FDMA does it after additional IDFT operation. Because of this difference, OFDMA is more sensitive to a null in the channel spectrum and it requires channel coding or power/rate control to overcome this deficiency. Also, the duration of the modulated time symbols are expanded in the case of OFDMA with parallel transmission of the data block during the elongated time period whereas SC-FDMA modulated symbols are compressed into smaller chips with serial transmission of the data block, much like a direct sequence code division multiple access (DS-CDMA) system.

\subsection{Peak Power Characteristics of SC-FDMA}

PAPR is a performance measurement that is indicative of the power efficiency of the transmitter. In case of an ideal linear power amplifier where we achieve linear amplification up to the saturation point, we reach the maximum power efficiency when the amplifier is operating at the saturation point. A positive PAPR in $\mathrm{dB}$ means that we need a power backoff to operate in the linear region of the power amplifier and high PAPR degrades the transmit power efficiency performance.

Unlike OFDM, statistical properties of PAPR for single carrier modulations are not easily obtained analytically [10]. We thus resort to numerical analysis to investigate the PAPR properties.

Figure 5 is the result of Monte Carlo simulations [11], it is the CCDF (Complementary Cumulative Distribution Function) of PAPR, which is the probability that PAPR is higher 
than a certain PAPR value PAPR0 (Pr $\{$ PAPR $>$ PAPR0 $\}$ ). We can see that all the cases for SC-FDMA have indeed lower PAPR than that of OFDMA. Also, IFDMA has the lowest PAPR, and DFDMA and LFDMA have very similar levels of PAPR.

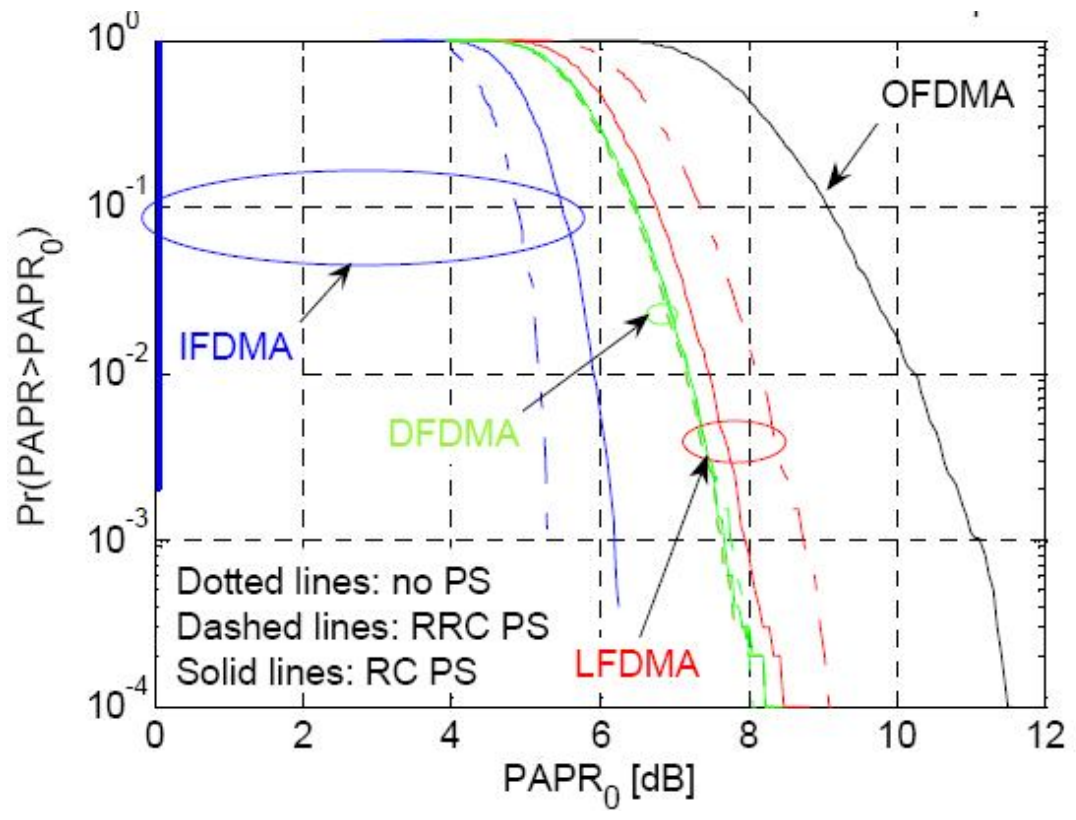

Fig. 5 Comparison of CCDF of PAPR for IFDMA, DFDMA, LFDMA, and OFDMA with total number of subcarriers $M=512$, number of input symbols $N=128$, IFDMA spreading factor 4, DFDMA spreading factor 2 , QPSK, and $\alpha($ roll-off factor $)=0.22$

If we apply IFDMA on a different number of subcarriers we observe that better PAPR reductions performance can be achieved for a large number of subcarriers. The PAPR results for the IFDMA and OFDMA systems are shown in Fig. 6. We can clearly see that as N increases PAPR results of OFDMA will get worse, while PAPR results of IFDMA will get better.

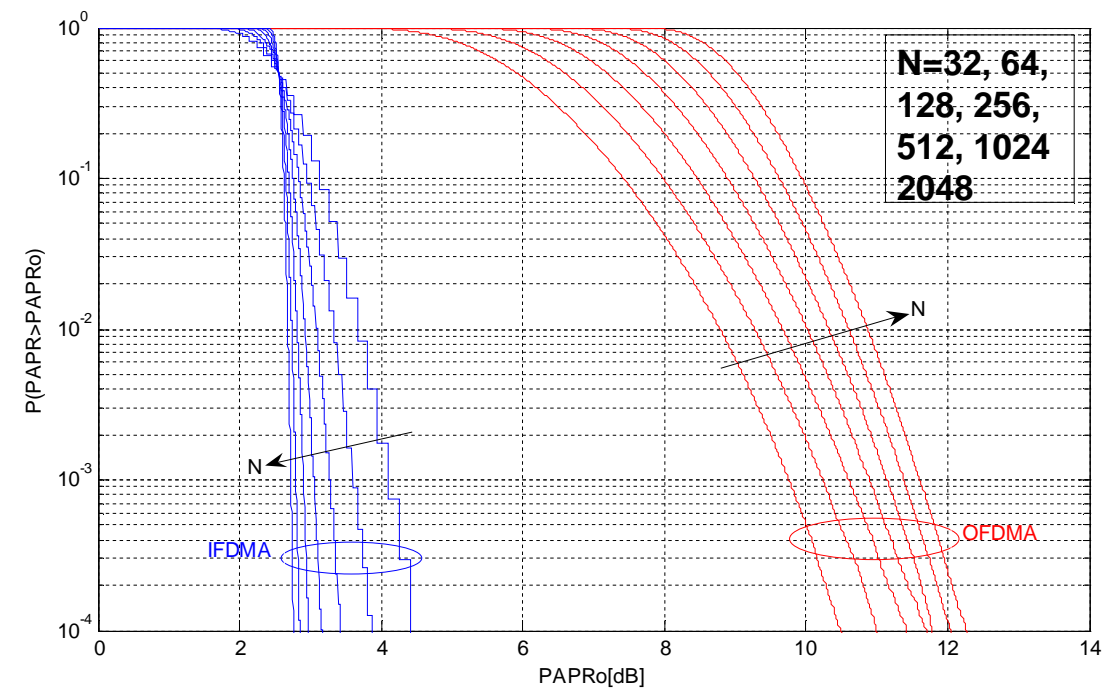

Fig. 6 PAPR results for IFDMA and OFDMA systems applied to a 16-QAM signal with various $N$ 
For example increasing $\mathrm{N}$ from 32 to 2048 , at a $10^{-4}$ clip probability, the PAPR in IFDMA systems can be reduced by $1.665 \mathrm{~dB}$, while the PAPR in OFDMA systems will be increased by $1.77 \mathrm{~dB}$. The results show that an IFDMA system can maintain a low PAPR even for a very large number of subcarriers. Inspired by this observation, for those OFDMA systems with limited subcarriers, we can first combine B frames into one super frame. Then use IFDMA with this super frame to reduce the PAPR.

Finally the super frame will be divided back into B frames. In this way, the PAPR can be reduced without requiring side information. The scheme proposed here is called IFDMA with super frame (SF-IFDMA). Instead of partitioning a frame into sub-blocks as in the partial transmit sequences (PTS) scheme, our scheme combines frames into one super frame.

\section{SC-FDMA with Super Frame Scheme}

In this section, the transmitter structure of the proposed scheme will be described first, and then the reduction in the PAPR of the proposed scheme will be presented. Figure 7 is the structure of the transmitter using super frame scheme. In the dashed box of Fig. 7 the consecutive $B$ transferred frames $x_{n}=\left[x_{n}(0), x_{n}(1), x_{n}(N-1)\right](n=1,2, \ldots B)$ in time domain are formed into one super frame, $x^{s}$ with N.B symbols. Then, the system performs DFT to $\mathrm{x}^{\mathrm{s}}$ to obtain a super frame $X_{\mathrm{k}}^{\mathrm{s}}$ with N.B symbols and maps each of the N.B-DFT outputs to one of the $\mathrm{M}(>N . \mathrm{B})$ orthogonal subcarriers $(M=Q . N . B)$ using interleaved mapping to produce $\widetilde{X}_{1}^{s}$. As in OFDMA, an M-point IDFT transforms the subcarrier amplitudes to a complex time domain signal $\mathrm{x}_{\mathrm{m}}^{\mathrm{s}}$. Then the super frame with lowered PAPR is divided back into $B$ frames $\widetilde{\mathrm{x}}_{\mathrm{m}}=\left[\tilde{\mathrm{x}}_{\mathrm{m}}(0), \tilde{\mathrm{x}}_{\mathrm{m}}(1), \tilde{\mathrm{x}}_{\mathrm{m}}(\mathrm{N}-1)\right](\mathrm{m}=1,2, \ldots \mathrm{B})$. Each of the final B SC-OFDM frames will be transmitted with their own guard intervals (GI) or training sequences (TS).

When the super frame with size N.B is divided back into B frames with size $\mathrm{N}$, the carrier spacing of final divided frame should remain the same as that of the original frame with $\mathrm{N}$ subcarriers.

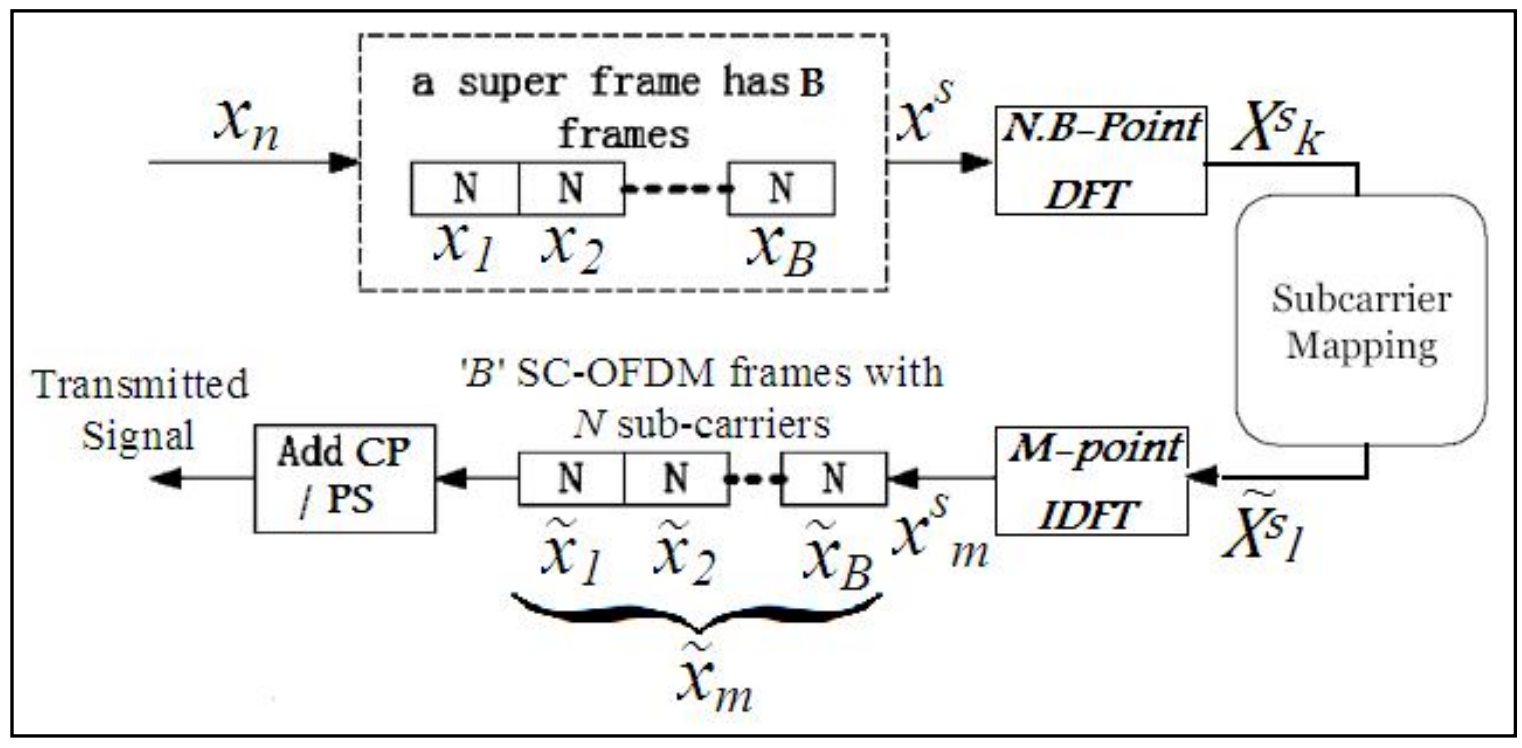

Fig. 7 The structure of the transmitter using SC-OFDM with super frame scheme 
For IFDMA, the frequency samples after subcarrier mapping $\widetilde{X}_{1}^{s}$ can be described as follows.

$$
\widetilde{X}_{1}^{S}=\left\{\begin{array}{cc}
X_{1}^{S} / Q & , l=Q . k(0 \leq k \leq N . B-1) \\
0 & , \text { otherwise }
\end{array}\right.
$$

We derive the time symbols $\mathrm{x}_{\mathrm{m}}^{\mathrm{s}}$ which are obtained by taking inverse DFT of $\widetilde{X}_{1}^{\mathrm{s}}$.

Let $\mathrm{m}=\mathrm{N}$.B. $\mathrm{q}+\mathrm{n}$, where $0 \leq \mathrm{q} \leq \mathrm{Q}-1$ and $0 \leq \mathrm{n} \leq \mathrm{N} \cdot \mathrm{B}-1$ then,

$$
\begin{aligned}
& \tilde{\mathrm{X}}_{\mathrm{m}}\left(=\mathrm{X}_{\text {N.B.q }+n}\right)=\frac{1}{\mathrm{M}} \sum_{\mathrm{l}=0}^{\mathrm{M}-1} \widetilde{\mathrm{X}}_{\mathrm{l}}^{\mathrm{s}} \mathrm{e}^{\mathrm{j} 2 \pi \frac{\mathrm{m}}{\mathrm{M}} \mathrm{l}}=\frac{1}{\mathrm{Q}} \cdot \frac{1}{\mathrm{~N} \cdot \mathrm{B}} \sum_{\mathrm{k}=0}^{\mathrm{N} \cdot \mathrm{B}-1} \mathrm{X}_{\mathrm{k}}^{\mathrm{S}} \mathrm{e}^{\mathrm{j} 2 \pi \frac{\mathrm{m}}{\mathrm{Q} \cdot \mathrm{N} \cdot \mathrm{B}} \mathrm{Q} \cdot \mathrm{k}} \\
& =\frac{1}{Q} \cdot \frac{1}{N \cdot B} \sum_{k=0}^{N \cdot B-1} X_{k}^{S} e^{j 2 \pi \frac{N \cdot B \cdot q+n}{N \cdot B}} \\
& =\frac{1}{Q}\left[\frac{1}{N \cdot B} \sum_{k=0}^{N \cdot B-1} X_{k}^{S} e^{j 2 \pi \frac{N \cdot B q}{N \cdot B} k^{j}} e^{j 2 \pi \frac{n}{N \cdot B}} k\right] \\
& =\frac{1}{\mathrm{Q}}\left[\frac{1}{\mathrm{~N} \cdot \mathrm{B}} \sum_{\mathrm{k}=0}^{\mathrm{N} \cdot \mathrm{B}-1} \mathrm{X}_{\mathrm{k}}^{\mathrm{S}} \mathrm{e}^{\mathrm{j} 2 \pi \frac{\mathrm{n}}{\mathrm{N} \cdot \mathrm{B}} \mathrm{k}}\right] \\
& =\frac{1}{\mathrm{Q}} \operatorname{IDFT}\left\{\mathrm{X}_{\mathrm{k}}^{\mathrm{S}}\right\}=\frac{1}{\mathrm{Q}} \mathrm{X}_{\mathrm{n}}
\end{aligned}
$$

The resulting time symbols $\tilde{\mathrm{x}}_{\mathrm{m}}$ are simply a repetition of the original input symbols $\mathrm{x}_{\mathrm{n}}$ with a scaling factor of 1/Q and some phase rotation in the time domain [9]. Therefore, the PAPR of SF-IFDMA signal is the same as in the case of conventional single carrier signal.

After the above process in Fig. 7, the PAPR of IFDMA signals can be effectively reduced. The use of a super frame allows the DFT and the IDFT to represent $x_{K}^{S}$ and $x_{m}^{s}$ respectively in a more accurate representation form. This form makes the output of the IDFT have less PAPR. The proposed scheme works even better when $N$ is small. In case that $N$ is already large enough, the proposed scheme has little improvement on PAPR reduction. From the results in Fig. 6, we can conclude that the proposed scheme is applicable to small $N$ with $N \leq 512$.

Compared with the traditional IFDMA system, the complexity of our proposed scheme does increase. At transmitter, the complexity increases due to the large number of symbols. At receiver, the same complexity is presented, as well as some delay introduced from receiving the "B" IFDMA frames before starting in the demodulation process.

\section{Simulation Results}

To show the overall performance of the proposed IFDMA with super frame scheme on reducing PAPR, we assume that randomly generated data are16-QAM modulated and the number of subcarriers is $\mathrm{N}=64$ and 128 respectively, with $\mathrm{B}=4$. Figure 8 gives the 
comparison of complementary cumulative function $(\mathrm{CCDF}), \mathrm{P}\left(\mathrm{PAPR}>\mathrm{PAPR}_{0}\right)$ between IFDMA and the proposed scheme. Figure 8 indicates that at given $C C D F=10^{-4}$, the PAPR of IFDMA signal with $\mathrm{N}=64$ is $3.87 \mathrm{~dB}$, while it is $3.14 \mathrm{~dB}$ under our proposed scheme. Our scheme has $0.7 \mathrm{~dB}$ improvement over IFDMA to reduce PAPR.

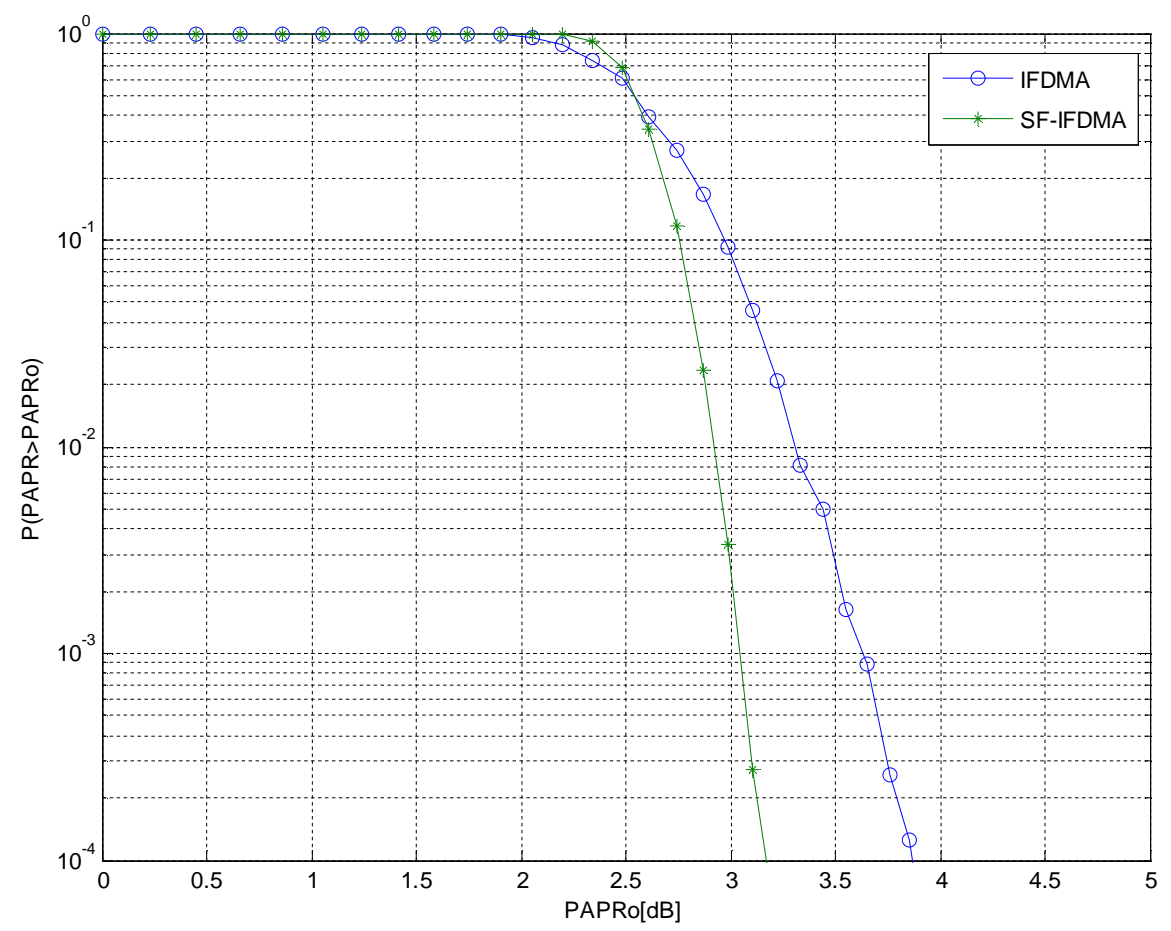

Fig. 8 CCDF for IFDMA and the proposed scheme with $N=64$ and $B=4$

Figure 9 is the CCDF, $\mathrm{P}\left(\mathrm{PAPR}>\mathrm{PAPR}_{0}\right)$ for IFDMA and the proposed scheme with $\mathrm{N}=128$. Figure 9 indicates that at given $\mathrm{CCDF}=10^{-4}$, the PAPR of IFDMA signal with $\mathrm{N}=128$ was $3.42 \mathrm{~dB}$, while it is $2.97 \mathrm{~dB}$ under our proposal. Our scheme has about $0.44 \mathrm{~dB}$ improvement in PAPR reduction. 


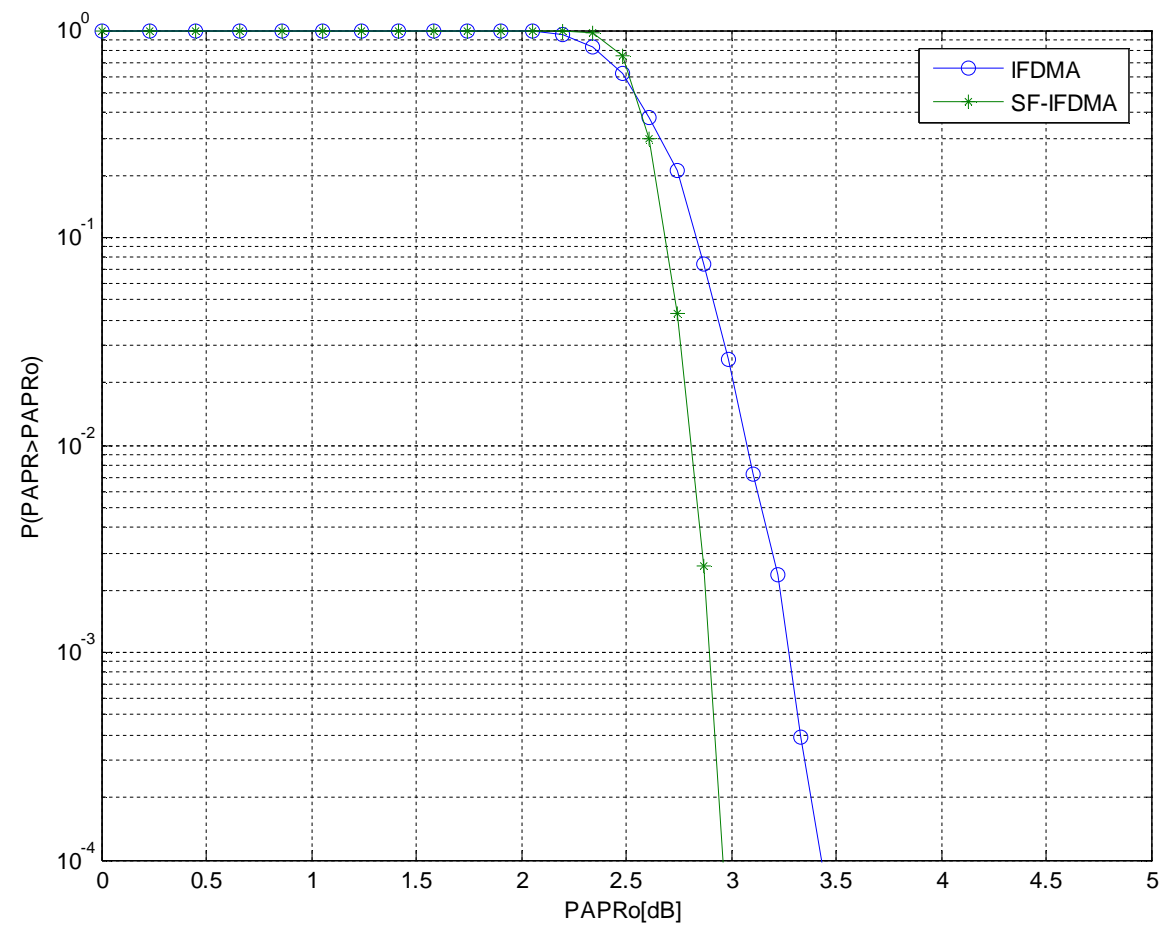

Fig. 9 CCDF for IFDMA algorithm and the proposed scheme with $N=128$ and $B=4$

Figures 10, 11 are the CCDF, $\mathrm{P}\left(\mathrm{PAPR}>\mathrm{PAPR}_{0}\right)$ for IFDMA and the proposed scheme with $\mathrm{B}=2,4,8,16$. Figures 10,11 indicate that at given $N=64,128$ respectively, there is no more improvement in PAPR of IFDMA signal with B $>4$.

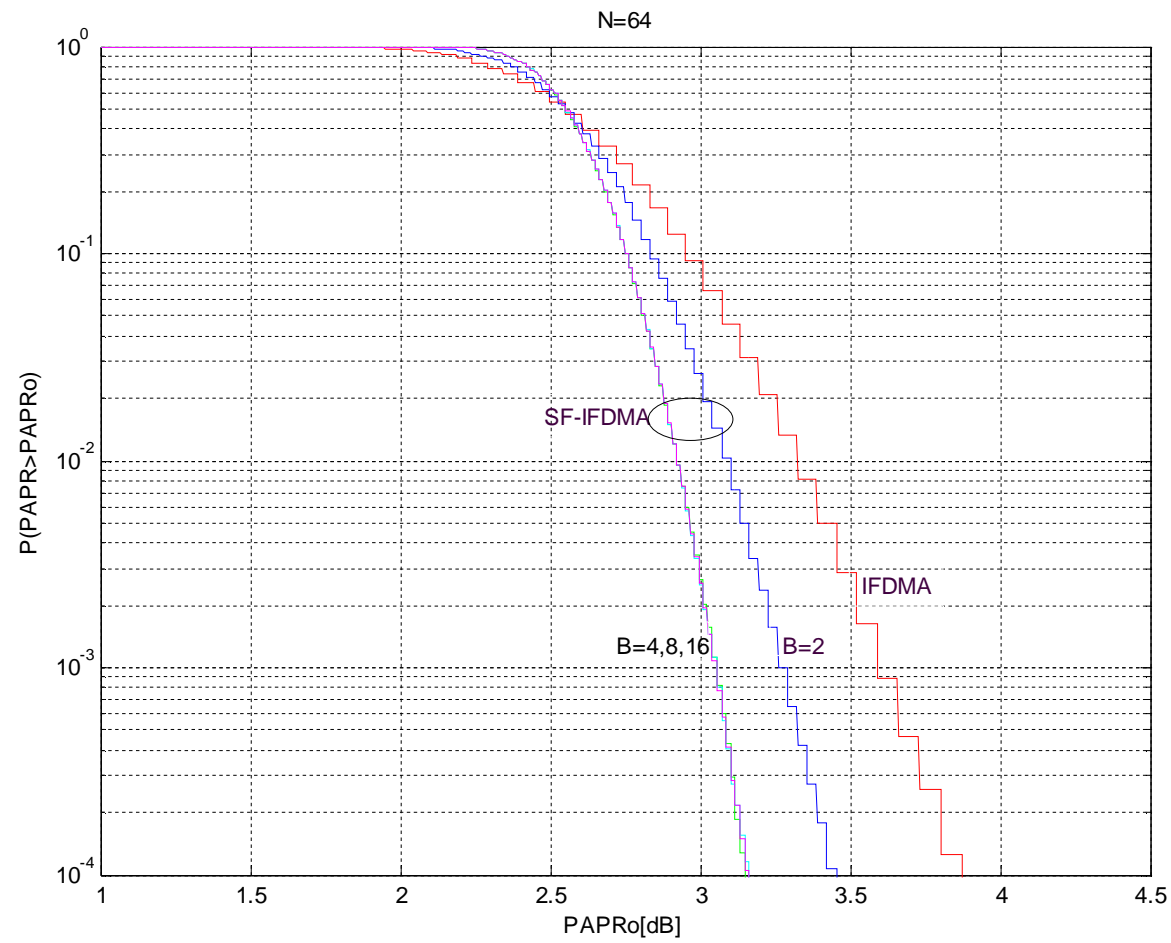

Fig. 10 CCDF for IFDMA algorithm and the proposed scheme with $N=64$ and $B=2,4,8,16$ 


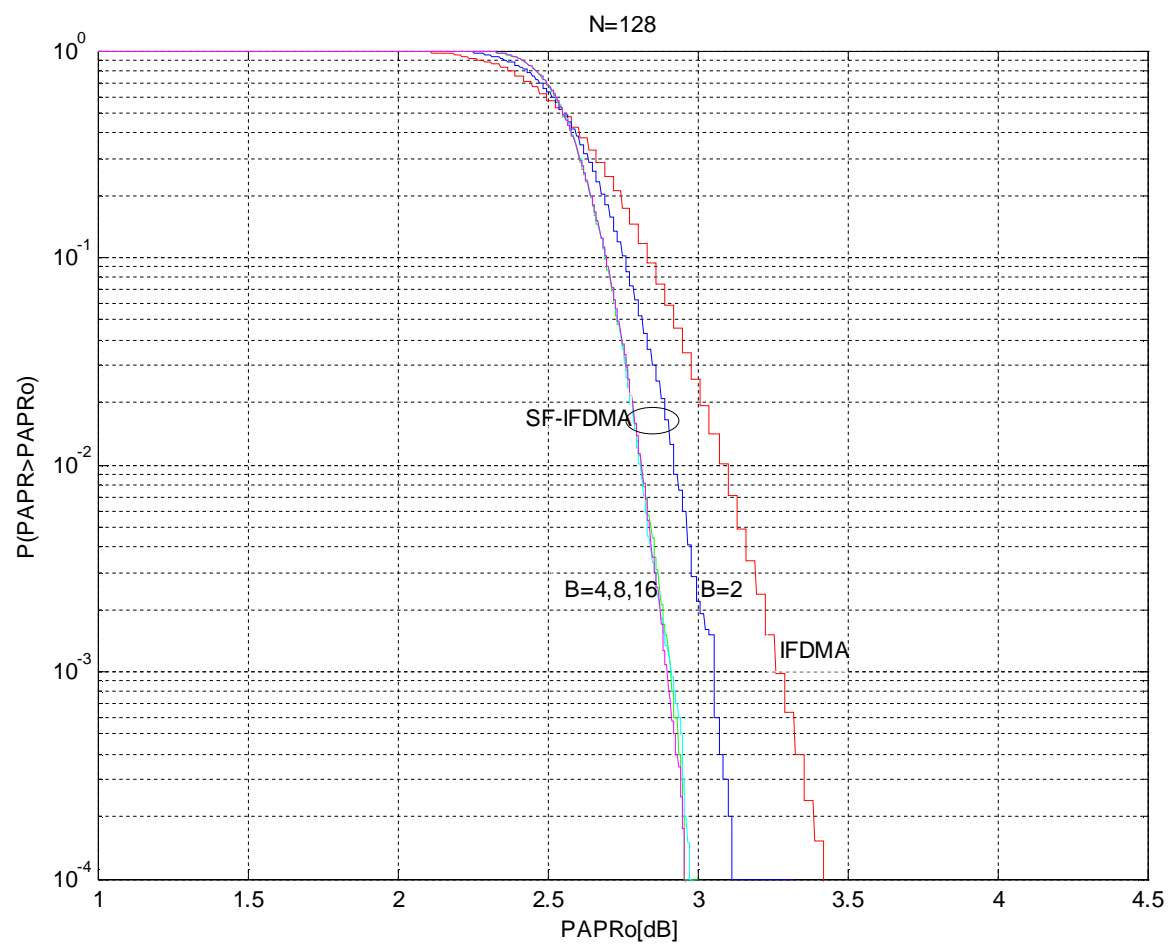

Fig. 11 CCDF for IFDMA algorithm and the proposed scheme with $\mathrm{N}=128$ and $\mathrm{B}=\mathbf{2 , 4 , 8 , 1 6}$

\section{Conclusion}

In this paper, we proposed a new scheme called IFDMA with super frame (SF-IFDMA), most suitable for the IFDMA systems with small number of subcarriers $\mathrm{N}$, for example $N \leq 512$. The novel scheme is very effective in reducing the PAPR in IFDMA systems, especially when $\mathrm{N}$ is small. It can achieve significant reduction of PAPR without requirement of any side information. This reduction comes at the price of some system complexity as well as some delay introduced from waiting the reception of "B" IFDMA frames at the receiver before starting in the demodulation process.

\section{References}

[1] R. van Nee and R. Prasad, OFDM for Wireless Multimedia Communications, Artech House, 2000.

[2] H. Sari, G. Karam,and I. Jeanclaude, "Transmission Techniques for Digital Terrestrial TV Broadcasting," IEEE Commun. Mag., vol. 33, no. 2, pp. 100-109, Feb. 1995.

[3] D. Falconer, S. L. Ariyarisitakul, A. Benyamin-Seeyar, and B. Eidson, "Frequency Domain Equalization for Single- Carrier Broadband Wireless Systems," IEEE Commun. Mag., vol. 40, no. 4, pp. 58-66, Apr. 2002.

[4] H. G. Myung, J. Lim, and D. J. Goodman, "Single Carrier FDMA for Uplink Wireless Transmission," IEEE Vehicular Technology Mag., vol. 1, no. 3, pp. 30 - 38, Sep. 2006.

[5] H. G. Myung, J. Lim, and D. J. Goodman, "Peak-to average Power Ratio of Single Carrier FDMA Signals with Pulse Shaping", Proc. IEEE International Symposium on Personal, Indoor and Mobile Radio Communications (PIMRC) 2006, Sep. 2006. 
[6] U. Sorger, I. De Broeck, and M. Schnell, "Interleaved FDMA - A New Spread Spectrum Multiple-Access Scheme," Proc. IEEE ICC '98, Atlanta, GA, pp. 1013-1017, Jun. 1998.

[7] M. Schnell and I. De Broeck, "Application of IFDMA to Mobile Radio Transmission," Proc. IEEE 1998 International Conference on Universal Personal Communications (ICUPC '98), Florence, Italy, pp. 1267-1272, Oct. 1998.

[8] H. G. Myung, "Single Carrier Orthogonal Multiple Access Technique for Broadband Wireless Communications," Ph.D. Dissertation, Polytechnic University, Brooklyn, NY, Jan. 2007.

[9] U. Sorger, I. De Broeck and M. Schnell, "Interleaved FDMA - A New Spread-Spectrum Multiple-Access Scheme,” Proc. IEEE ICC '98, pp. 1013-1017, Atlanta, GA, June 1998.

[10] D. Wulich and L. Goldfeld, "Bound of the Distribution of Instantaneous Power in Single Carrier Modulation," IEEE Trans. Wireless Commun., vol. 4, no. 4, pp. 17731778, Jul. 2005.

[11] Hyung G. Myung, Introduction to Single Carrier FDMA, EURASIP, 2007. 